\title{
Apoptosis Pathways in Ovarian Cancer
}

\section{Introduction}

Christine Sers ${ }^{1}$, Reinhold Schafer ${ }^{1}$ and Irina Nazarenko ${ }^{2}$

${ }^{1}$ Institute of Pathology,

University Medicine Charité, Berlin

2Institute of Toxicology and Genetics, Karlsruhe Institute of Technology, Karlsruhe

Germany

Tumour initiation and progression are driven by constitutively activated oncogenes mediating deregulation of the balance between cell death- and survival pathways. Among the most relevant signalling cascades activated in the majority of tumour types, the RAS/mitogen-activated protein kinase (Ras/MAPK), the phosphatidyl inositol-3kinase/protein kinase B (PI3K/PKB) and the protein kinases $\mathrm{C}$ (PKC) signalling cascades were postulated (Weinstein, 1987; Nicosia et al., 2003; Roberts and Der, 2007; McCubrey et al., 2007; Breitkreutz et al., 2007). These cascades define individual characteristics of particular tumours and consequently their individual responsiveness to cancer therapy.

In this chapter, we will address the characteristics of the apoptotic signalling pathways in ovarian carcinomas. Particular attention will be given to the HRS family of tumour suppressor genes encoding proteins with phospholipase activity and suppressed in the majority of ovarian malignancies. We will describe signalling cascades down regulating two well-characterized members of this family H-REV107-1/HRLS3/PLA2G16 and TIG3/RARRES/RIG1 in tumour cells. Furthermore, potential therapeutic consequences of the re-expression of these genes defined as a class II tumour suppressors will be discussed.

\section{The HRS class II tumour suppressors are important mediators of IFN- $\gamma$ and retinoid-dependent growth suppression and cell death in ovarian cancer}

The H-REV107-related genes (TIG3, H-REV107-1, HRSL2) are known as inhibitors of proliferation of tumour cells in vivo and in vitro. While being almost ubiquitously expressed in normal tissues, down-regulation or complete loss of these genes in tumours and tumour cell lines have been reported. Expression can be reconstituted by different anti-proliferative signals such as interferons and retinoids, as well as by the inhibition of oncogenic pathways and interference with DNA methylation (Alessi et al., 1994; Husmann et al., 1998; Akiyama et al., 1999; Siegrist et al., 2001; Ito et al., 2001; Roder et al., 2002; Huang et al., 2002; Higuchi et al., 2003; Duvic et al., 2003). Re-activation of the H-REV107-1-related proteins and overexpression of the genes induce apoptosis or differentiation of tumour cells. 


\subsection{HRS family members encode LRAT-related phospholipid-metabolizing enzymes}

Two independent groups (Hughes and Stanway, 2000; Anantharaman and Aravind, 2003) have unfolded the phylogenetic relationship between H-REV107-1-related genes, LRAT (Lecithin retinol acyltransferase) and viral and bacterial peptidases in previous works.

Here we aimed to identify and describe H-REV107-1 homologs in different organisms in order to follow their origin and development during the evolution.

For that purpose, we performed an in silico analysis, using PSI-Blast and Blast-p screening in the NCBI non-redundant database. This analysis revealed 62 homologous proteins in eukaryotic and prokaryotic organisms. To identify phylogenetic relationships Tree Puzzle (Strimmer and von Haeseler, 1997) was applied (Fig. 1). The analysis revealed five closely related proteins, suggesting their origin from the same ancestor protein during the evolution. These proteins, including H-REV107-1 comprise a new protein family, which we designated here as the HRS (H-REV107-1-related proteins) protein family (Table 1).

The novel HRS family is composed of tumour suppressors, which negatively regulate cell survival, control signal transduction and induce differentiation.

\begin{tabular}{|c|c|c|c|c|c|c|c|}
\hline $\begin{array}{l}\text { Suggested } \\
\text { nomenklature }\end{array}$ & NCBI Synonyms & $\begin{array}{l}\text { Chromosomal } \\
\text { localization }\end{array}$ & Species & Publication - gene cloning & Acc. No gene & Acc. No protein & Function \\
\hline HRS1 & HRASLS & $\begin{array}{c}3 \mathrm{q} 29 \\
(194440-1944470 \\
\mathrm{K})\end{array}$ & Human & $\begin{array}{l}\text { Ito et a1. Cytogenet. Cell } \\
\text { Genet. } 93: 36-39(2001)\end{array}$ & NM_020386 & NP_065119 & Unknown \\
\hline Hrs1 & $\begin{array}{c}\text { A-C1, Hras1s, } \\
\text { 2810012B06Rik }\end{array}$ & 16 & Mouse & $\begin{array}{c}\text { Akiyama et al. J. Biol. Chem. } \\
\text { 274: } 32192-32197(1999)\end{array}$ & NM_013751 & NP_038779 & $\begin{array}{l}\text { Inhibits growth of HRAS- } \\
\text { transformed cells }\end{array}$ \\
\hline Hrs1 & similar to Hrasis & & Rat & \multirow{2}{*}{$\begin{array}{c}\text { Uyama et al., } \\
\text { Biochim.Biophys.Acta (2009) }\end{array}$} & $X \mathrm{X} \_213590$ & XP_213590 & \multirow{2}{*}{$\begin{array}{l}\text { PE } N \text {-acyltransferase } \\
\text { lysophospholipid } O- \\
\text { acyltransferase }\end{array}$} \\
\hline HRS2 & HRASLS2 & $\begin{array}{c}11 q 12.3(63076- \\
63088 \mathrm{~K})\end{array}$ & Human & & NM_017878 & NP_060348 & \\
\hline HRS3 & $\begin{array}{l}\text { HRASLS3, } \\
\text { H-REV107-1, } \\
\text { H-REV107-3, } \\
\text { hHrev-107, } \\
\text { PLA2G16 }\end{array}$ & $\begin{array}{c}11 q 12.3-q 13.1 \\
(63099-63138 \mathrm{~K})\end{array}$ & Human & $\begin{array}{l}\text { Husmann et al. Oncogene } \\
\text { 17: 1305-1312(1998) }\end{array}$ & NM_007069 & NP_009000 & $\begin{array}{l}\text { Phospholipase } 2 \mathrm{~A} \text {, } \\
\text { Induces apoptosis in } \\
\text { ovarian cancer cells }\end{array}$ \\
\hline Hrs3 & Hrasis3 & 19 & Mouse & $\begin{array}{l}\text { Roder et a1. J: Biol. Chem. } \\
\text { 277: } 30543-30550(2002) ; \\
\text { Jaworski et a1., Nat Med. } \\
2009\end{array}$ & NM_139269 & NP_644675 & $\begin{array}{l}\text { major adipocyte } \\
\text { phosphoslipase }\end{array}$ \\
\hline Hrs3 & $\begin{array}{l}\text { Hras1s3, } \\
\text { H-Rev107 }\end{array}$ & $1 \mathrm{q}$ & Rat & $\begin{array}{l}\text { Hajnal et al. Oncogene 9: } \\
\qquad 479-490(1994)\end{array}$ & NM_017060 & NP_056756 & $\begin{array}{l}\text { Inhibits growth of Ras- } \\
\text { transformed cells in vivo } \\
\text { and in vitro }\end{array}$ \\
\hline HRS4 & $\begin{array}{l}\text { RARRES3, } \\
\text { TIG3, RIG1, } \\
\text { H-REV107-2 }\end{array}$ & $\begin{array}{l}11 \mathrm{q} 13.2(63061- \\
63070 \mathrm{~K})\end{array}$ & Human & $\begin{array}{l}\text { DiSepio et a1. PNAS 95: } \\
\text { 14811-14815(1998) }\end{array}$ & NM_004585 & NP_004576 & $\begin{array}{l}\text { phospholipid } \\
\text { methabolizing enzyme, } \\
\text { Induces apoptosis in } \\
\text { ovarian cancer cells }\end{array}$ \\
\hline HRS5 & HRLP5; iNAT & $\begin{array}{c}11 q 13.2(62988- \\
63015 \mathrm{~K})\end{array}$ & Human & \multirow{6}{*}{$\begin{array}{c}\text { Jin et al., } \\
\text { Biochim.Biophys.Acta } \\
\text { (2009); Jin et a1., J.Biol. } \\
\text { Chem (2007) }\end{array}$} & NM_054108 & NP_473449 & \multirow{6}{*}{$\begin{array}{c}\text { Ca-independent } \\
\text { phosphatidylethanolamine } \\
\text { N-acyltransferase }\end{array}$} \\
\hline Hrs5 & Hrlp5 & & Mouse & & NM_025731 & NP_080007 & \\
\hline Hrs5 & Hrlp5 & & Rat & & XM219546 & XP_219546 & \\
\hline Hrs & unnamed & & Fish & & CAG09755 & CAAE01015008 & \\
\hline Hrs & MGC68773 & & Frog & & BCO60489 & AAH60489 & \\
\hline Hrs & unnamed & & Amphioxus & & AF391288 & AAM18866 & \\
\hline
\end{tabular}

Table 1. Members of the HRS family 


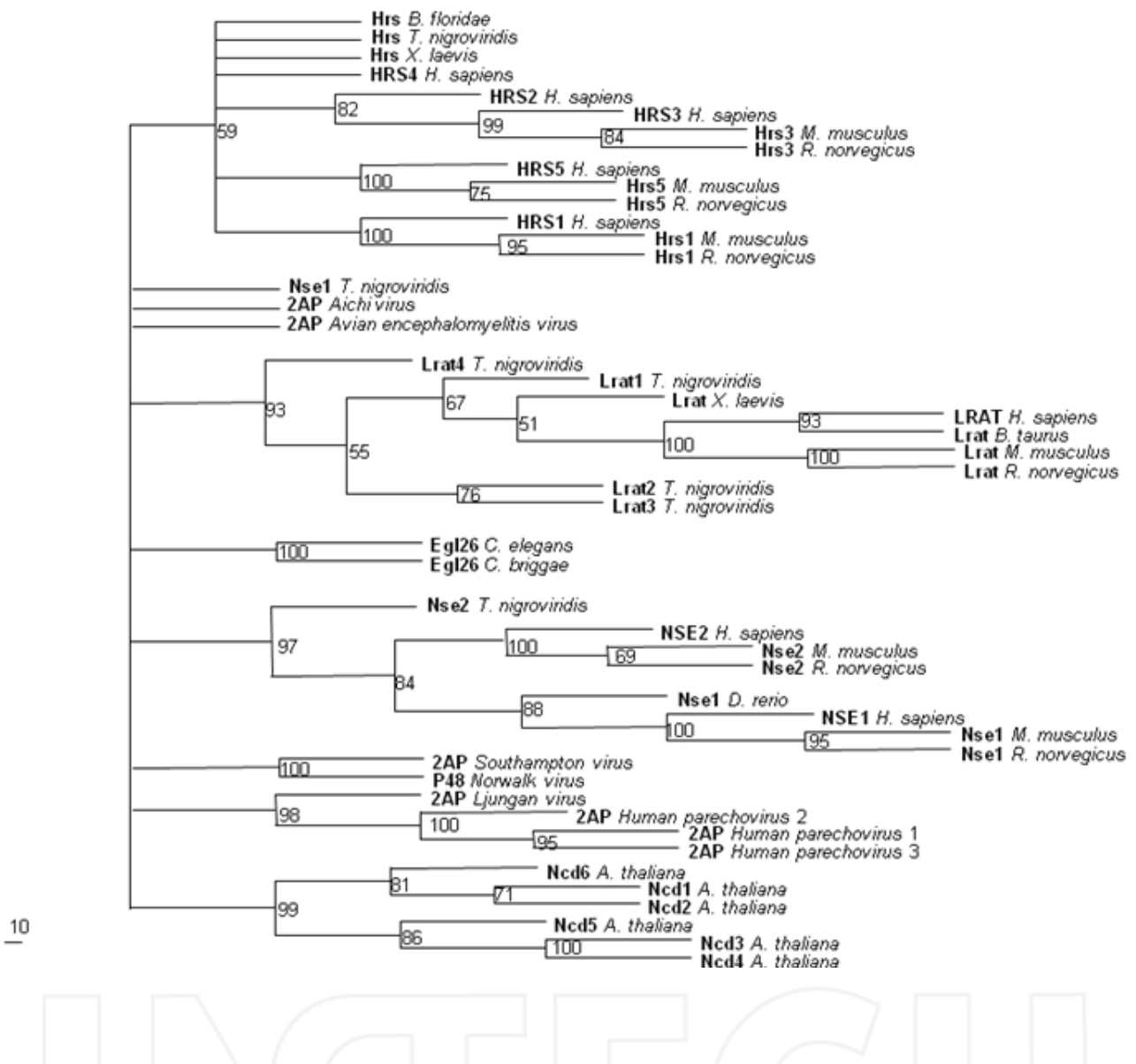

Fig. 1. Phylogeny of HRS and the related LRAT, NSE and NCD protein families A maximum parsimony tree was generated with the help of Tree-Puzzle (Strimmer and von Haeseler 1997) and includes the eukaryotic members of the HRS, LRAT, NSE families, plant NC proteins, the C.elegans Egl26 proteins, and the viral 2A proteins.

First, only HRS proteins were aligned using the ClustlW algorithm (EMBL-EBI), then LRAT and NSE families. From the C.elegans, the viral and the plant proteins only regions with a high similarity to the HRS proteins were compared. Additional upstream and downstream motifs were cut out. As a result, all sequences had a comparable length of about 160 amino acid residues that corresponds the average length of the HRS proteins.

For the calculation of the phylogenetic relationships 1000 replicates were run. Branch support values are indicated at the nodes, distances are proportional to relative sequence divergence. 


\subsection{Clustered chromosomal localization of 4 human HRS genes on 11q13}

Four of the five members of the human HRS family, HRLS2, H-REV107-1, TIG3 and HRLS5, are localized in one cluster on chromosome 11q13, supporting the hypothesis of their origin from the same ancestor (Fig. 3). The HRLS2 and H-REV107-1 genes, encoding most closely related family members (Fig. 1A), are located next to each other. The H-REV107-1 gene spans between $63099 \mathrm{~K}$ and $63138 \mathrm{~K}$ on the chromosome 11q, and directly downstream of it, from $63077 \mathrm{~K}$ to $63088 \mathrm{~K}$, the HRLS2 gene is located. The TIG3 gene is positioned on the opposite DNA strand directly downstream of HRLS2, the gene has a small non-coding region and spans between $63070 \mathrm{~K}$ and $63079 \mathrm{~K}$. The HRLS5 gene $62897 \mathrm{~K}$ and $63015 \mathrm{~K}$ is also located on chromosome 11q13, but separated by two genes encoding the thymosin-like 5 (TMSL5) and the lectin, galactoside-binding, soluble, 12 (galectin 12/LGAL12) proteins, from the other HRS genes.

Earlier findings suggested that chromosomal alterations resulting in HRS gene downregulation or loss are rather rare events in human carcinomas. Nevertheless, structural changes on 11q13 have been described in numerous cases and only recently methods such as array CGH and next generation sequencing (NGS) have improved the analysis such that the involvement of individual genes can now be analysed. Therefore, it cannot be excluded that future investigations might unravel smaller deletions influencing one of the clustered HRS genes on 11q13 in distinct tumour types.

\subsection{Domain structure and enzymatic activity of the HRS family members}

Phylogenetic analysis of HRS and HRS-related proteins revealed a high conservation within the so called NlpC/P60 domain (Anantharaman and Aravind, 2003). This sequence was indentified in LRAT proteins (lecithin retinol acyltransferase) as being essential for all-transretinol metabolism.

To analyse domain structure of other members of the HRS family, Clustl W alignment was performed. Using this program, 14 members of the HRS protein family found in the NCBI database, were analysed (Fig. 2).

The HRS proteins contain non-homologous proline-rich motifs on their N-termini (red line on the top of the alignment). The core parts of HRS proteins are highly conserved and contain the NlpC/P60 and NC domains ( Fig. 2, blue and green boxes, respectively). We predicted three $\beta$ strands within the Nlpc/P60 domain (Fig. 2, blue arrows). The first and the second strand contain the conserved GDL and HWXXY motifs; the VXXLAP motif comprises the third strand. The region downstream the third $\beta$-strand with two conserved serine residues is likely to have the structure of $\alpha$-helix ( Fig. 2, green cylinder). The large NC domain depicted in Fig. 2 with a green box, contains a KALVK conserved motif of unknown function, two short stretches DXXG and NKXD, which are similar to conserved regions of GTPases (Akiyama et al., 1999; Bourne, Sanders, and McCormick 1990) and the NCEHFV conserved motif, characteristic for conventional NC domains. At the C-terminus, HRS proteins harbour a hydrophobic C-terminal $\alpha$-helix,described as a membrane-binding domain.

Recently, a crystal structure of the NlpC/P60 domain of H-REV107-1 has being resolved (Ren et al., 2010b). Within this domain, a phospholipase active site consisting of a Cys-HisHis triad was identified. The residues $\mathrm{H} 23$ and C113 play a pivotal role for the H-REV107-1 enzymatic activity (Ren et al., 2010a). Meantime, the enzymatic activity of the H-REV107-1, TIG3, HRASLS2 and HRLP5 proteins has been characterized as PLA $1 / 2^{-}$type hydrolysis, supporting a role of the HRS proteins in lipid metabolism. 


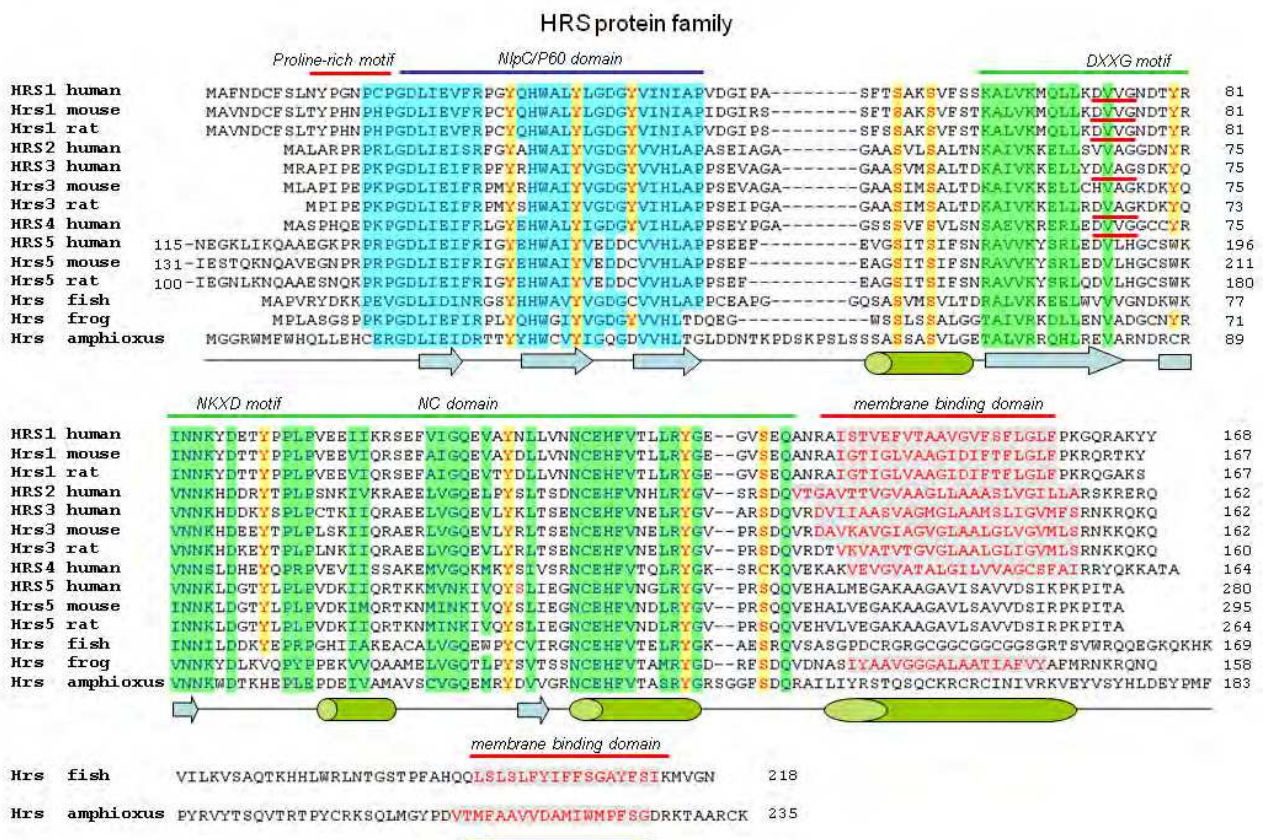

Fig. 2. HRS catalytic and protein-binding domains

Fourteen members of the HRS protein family found in the NCBI database were aligned using the Clustal W program as described in Figure 1B. Analysis, edition, and shading of conserved domains were performed with the help of the GenDoc freeware (http://www.psc.edu/biomed/genedoc/). The PSIPRED secondary structure prediction server was used to analyze potential secondary structures of the HRS protein sequences (http://bioinf.cs.ucl.ac.uk/psipred/) (McGuffin, Bryson, and Jones 2000).

The HRS proteins contain non-homologous proline-rich motifs on their N-termini (red line on the top of the alignment). The core parts of HRS proteins are highly conserved and contain the NlpC/P60 and NC domains (blue and green boxes, respectively). With a high prediction confidence of the PSIPRED standard analysis, three $\beta$-strands were defined within the Nlpc/P60 domain (blue arrows). The first and the second strand contain the conserved GDL and HWXXY motifs; the VXXLAP motif comprises the third strand. The region downstream the third $\beta$-strand with two conserved serine residues is likely to have a structure of $\alpha$-helix (green cylinder). The large NC domain (green box) contains a KALVK conserved motif of unknown function, two short stretches DXXG and NKXD, which are similar to conserved regions of GTPases (Akiyama et al., 1999; Bourne, Sanders, and McCormick 1990) and the NCEHFV conserved motif, characteristic for conventional NC domains. At the C-terminus, HRS proteins harbour a hydrophobic C-terminal $\alpha$-helix. 
HRS genes cluster

human chromosome 11 , region $62,975 \mathrm{~K}-63,147 \mathrm{~K}$ bp

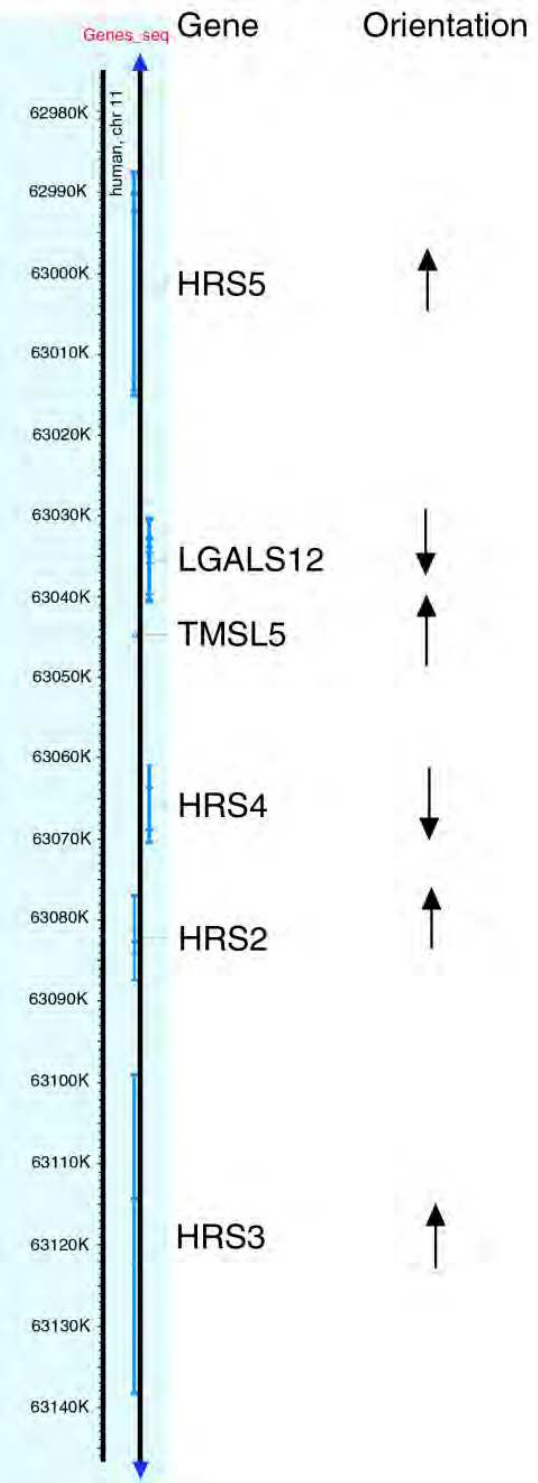

Fig. 3. HRS2/HRLS2, HRS3/H-REV107-1/ HRS4/TIG3 and HRS5/HRLS5 genes are localized on chromosome 11q13 in one cluster. Gene orientation, length and mapping of the chromosome regions are directly obtained from the NCBI Map View server.

Additionally to the NlpC/P60 domain, HRS proteins contain a proline-rich $\mathrm{N}$-terminal domain, responsible for establishing protein-protein interactions and a variable 
hydrophobic C-terminal $\alpha$-helix, which directs and transiently binds the protein to intracellular membranes (Husmann et al., 1998; Nazarenko et al., 2007). Furthermore, a DXXG domain, also termed G3 motif, characteristic for RAS small GTPases, and mediating the binding of magnesium and $\gamma$-phosphate of GTP via the aspartic acid and glycine residue, respectively was identified (Kjeldgaard et al., 1996). However, a functional role of these motifs in HRS proteins has not been defined yet.

\subsection{Reversible Inhibition of the HRS genes H-REV107-1 and TIG3 by oncogenic signalling cascades in tumours}

Members of the HRS gene family H-REV107-1 and TIG3 belong to the so called class II tumour suppressors. The major characteristic of this class, postulated in 1997 by Ruth Sager, is their down-regulation in tumours via reversible mechanisms, however not through mutations or deletions (Sager, 1997). Once re-expressed, these genes can exhibit their tumour-suppressive function and thereby contribute to the inhibition of tumour progression.

\subsubsection{IFN $\gamma$-mediated re-expression of H-REV107-1 leads to the induction of apoptosis in ovarian cancer cells}

Rat H-Rev107-1, the founder gene of the HRS family, was cloned from a subtractive cDNA library (Hajnal et al., 1994). The rat H-Rev107-1 gene, expressed in immortalized rat fibroblasts, was identified as a gene suppressed in an HRAS-transformed derivative, but reexpressed in a revertant cell line. Further experiments suggested that repression of $\mathrm{H}$ Rev107-1 in HRAS-transformed cells was functionally involved in HRAS-dependent transformation (Hajnal et al., 1994; Sers et al., 1997).

Repression of H-Rev107-1 was also detected in KRAS-transformed rat ovarian epithelial cells suggesting that in contrast to other HRS genes, H-Rev107-1 suppression in response to RAS oncogenes is not associated with the RAS isoform. Most interestingly, H-Rev107-1 downregulation upon KRAS-transformation appeared to be reversible and identified the $\mathrm{H}$ Rev107-1 gene as a target negatively regulated by the MEK-ERK pathway. The same observation was also made in PA1 human teratocarcinoma cells, which harbour an activated NRAS oncogene (Alessi et al., 1994) and suggested that H-REV107-1 might be negatively affected by RAS oncogene-dependent signalling in general.

The human H-REV107-1, first described in 1998, was found ubiquitously expressed in normal human epithelial tissues (;Husmann et al., 1998). When compared to differentiated cells in situ, H-REV107-1 is down-regulated in human tumour cell lines and tumour samples at the mRNA and at the protein level. Loss of H-REV107-1 mRNA until now was detected in tumours derived from breast, lung, ovary, kidney and testis (Sers et al., 1997;Siegrist et al., 2001). In human ovarian carcinomas, we also demonstrated strongly diminished levels or complete loss of the H-REV107-1 protein. In ovarian carcinomas sequencing of the $\mathrm{H}$ REV107-1 coding region revealed no alterations within this region suggesting that $\mathrm{H}$ REV107-1 acts as class II tumour suppressor gene in these tissues.

A functional involvement of H-REV107-1 inactivation in ovarian tumours was demonstrated by the finding that reactivation of endogenous H-REV107-1 in H-REV107-1-negative ovarian carcinoma cells induces apoptosis. In these cells, loss of H-REV107-1 expression can be reconstituted upon administration of Interferon gamma (IFN $\gamma$ ), a finding reported earlier from rat astrocytoma cells (Bartel, 2004). Up-regulation of H-REV107-1 in response to IFN $\gamma$ 
works well at the mRNA level, yet only a small proportion of cells also express sufficient HREV107-1 protein for detection. Most interestingly these cells undergo apoptosis (Sers et al., 2002). These observations made clear that H-REV107-1 is likely to interfere with the survival of ovarian cancer cells. Our work further supported this suggestion as we could show that H-REV107-1 is an inhibitor of PP2A whose function is required in ovarian carcinomas for cell survival (Nazarenko et al., 2007). This was the first hint indicating a role of the $\mathrm{H}$ REV107-1 protein in the regulation of apoptotic intracellular signalling and will be discussed in part 3 of this chapter.

\subsubsection{Mechanisms of H-REV107-1 suppression in ovarian carcinomas via anti- apoptotic pathways}

The reversible down-regulation of H-REV107-1 in ovarian cancer has prompted the investigation of the mechanisms responsible for suppression. The human H-REV107-1 promoter is located directly upstream of a $408 \mathrm{bp} 5^{\prime} \mathrm{UT}$ sequence. The sequence harbours several potential transcription factor binding sites including an Interferon-responsive IRSE motif, a CREB site, potential AP-1 and c-REL binding sites (Fig. 4).

The IRSE site, a DNA-sequence bound by the Interferon regulatory factors IRF-1 and IRF-2, provides the structural basis for the observed induction of H-REV107-1 upon administration of IFN $\gamma$ and conditionally expressed IRF-1 (Alessi et al., 1994). Comparison of IRF-1 and $\mathrm{H}$ REV107-1 levels between human ovarian carcinoma cells and immortalized human ovarian epithelial cells, revealed strongly diminished IRF-1 and H-REV1017-1 levels in the tumour cell lines. This suggested that loss of IRF-1 expression might be one of the mechanisms of $H$ REV107-1 suppression in human ovarian carcinomas (Sers at al., 2002).

Surprisingly, there is no conservation between the human H-REV107-1 and the mouse or rat H-REV107-1 promoter region, suggesting a different regulation of human H-REV107-1 and the rodent homologues. More importantly, it was shown that murine H-REV107-1 can be regulated via DNA methylation. In view of human tumours, next steps will include addressing the question, whether this methylation-dependent suppression of H-REV107-1 is a tumour-related process, or a developmental process during which tissue-specific expression profiles are established.

\subsubsection{Physiological role of H-REV107-1 and its potential role in cancerogenesis}

Meantime, the enzymatic function of H-REV107-1 has been defined (Ueda et al., 2009). The protein acts as a cytosolic $\mathrm{Ca}^{2+}$-independent phospholipase Pla2G16, which catalyses esterolytic cleavage of glycerophospholipids to lysophospholipids. Supporting these data, a recent study in a knock-out model demonstrated that the H-rev107-1 physiological function is a major adipocyte phospholipase $\mathrm{A}_{2}$ (AdPLA). The protein inhibited lipolysis in adipocytes, regulating adiposity on systemic level (Jaworski et al., 2009). Ablation of the Hrev107-1 led to a significantly higher rate of lipolysis, accompanied by an increase in cyclic AMP levels (Jaworski et al., 2009). The knock-out animals were resistant to high-fat feeding and leptin-deficiency mediated obesity. Albeit, a direct impact of the H-rev107-1 ablation on tumourigenesis in vivo has not been tested yet, the observed increase in lipolysis and elevated levels of cAMP, also common in tumour cells, suggest a potentially higher susceptibility of the H-rev107-1 knockout animals to tumour growth as compared to their wild type littermates. 
Promoter sequence of the H-REV107-1 gene

-997 TGAGACTGAGTTTCGCTTTGTTGCACAGGCTAGAGTGCAGTGGCGCGATC ISRE bzw.

IRF-1/IRF-2 Element

-947 TTGGCTCACTGCAACCCCCACCTCCCGGGCTCAAGTGATTCTCCTGCCTC

-897 AGCCTCCCGAGTAGCTGGGACTACAGGCGCATGCCTCCACGCCCGGCTAA

-847 TTTTTTGCATTTTTAG TAGAGACGGGGTTTCATOGTGTTAGCCAGCATGG

AP-4

-797 TCTCGATCTCTTGACCTOGTGATCTGCCCGCCTOGGCCTCCCAAAGTGCT AP-1

-747 GAGATTACAGGCGTGAGCCACOGCGCCCGGCCCTTGGTGGTATATTTTTA

-697 ACTCCTTCAGTTTTTAAACTA TAAGCCCATTCTTGAGTGAAGGCGAAAGT

-647 AAACCCATCATGGCCCTGCAGTGTGATGTGTGTGCAGAGGTCGAGTGTGT

-597 GCGACTCCTGGATGCTGGGCGCGCAGGGCATGGGTGAGGCGGGAAGAGGC

-547 GGTGCCGGGGGCGCGGGCGTCCTGCAGTCGCCGGGCTOGGGACCGGGGCC

-497 GGGCGCTCTGCGAGGCTCTCATTAGCCGGCGGCGCGGGGÄGGGCCGGGT

GC-Box

max. TSP :

-447 GACCTCACGCCGGCCCGOCACOGCGGCCÄTTAGACCCGGTCCAATTGCTG CREB-1

CCAAT-Box \#1 CCAAT-Box $\# 2$

-397 GGGCTGCAGCGCTGCCTOCGAGACCGCGAAGTGGGTGGATCGGGTCTTCC STAT-1

cRel \#1

-347 T̈GGÄÄGGTGCGATAAGGOCGGGCGAGGTGCCTGGGATGCTTCTCCCCTTC cRel t2

-297 CGCGAGGAAGAGATCTAATTGGGTAGGGCGGGTGTAGACTAGCCTGCCGA

-247 GCCGCCCGCTGGCACCTGCAGOCTCCTGGGCGCOCGCGGGCCCCGGCGAG

-197 AAAGTTGTTAAAGGGAGCGAGGTGGTTGTTCCTGGGGTACGAGGCGCGCC

-147 TCTCACGCCCTGCCCA.ACAGA.AGCCGCAGTCCGTGGGGTCTGGAGACGCA

- 97 GTTTCCTTGTTAATGACAATAAATCCCTGCTCCOCCTGCCTCAGACATCT

- 47 ACGCAGCGAAATCGAGCCTGGOCTTGAGGGTOCACACCGCGAGGA.AGATG

+4 CGTGCGCCCATT

Fig. 4. Promoter sequence of the human H-REV107-1 gene. The translatioal start site is indicated by $+1,997$ base pairs of upstream sequence are shown. Individual sequence motifs as identified by MatInspector are indicated. 
The H-rev107-1 knockout model provides a first link between lipid metabolism and a tumour suppressive effect of phospholipases. Alterations in lipid metabolism, especially in phospholipids-related pathways and fatty acid biosynthesis are known to occur in ovarian carcinomas (Tania et al., 2010). . Thus, FAS (fatty acid synthase) is up-regulated in cancer cells and mediates activity of HER-2 (Gansler at al., 1997; Menendez et al., 2004). It has been suggested that HER-2 functions as a cellular energy sensor in response to the metabolic stress, supporting therapeutic advantages of combinatorial inhibition of HER-2 and FAS in HER-2-positive tumours. However, phospholipases PLA2 were known to function as positive regulators of cell proliferation and migration (Song et al., 2007), playing rather a tumour-promoting role. In contrast to that, we and other uncovered a tumour-suppressive function of H-REV107-1 and its related proteins functioning as PLA2 enzymes.

It is likely that these observations provide a new link between malignant transformation, tumour progression and alteration in lipid metabolism, which needs further investigations. An important aspect needs to be refurbished according to the latest findings, is a change of lipid metabolic in tumour-surrounding stroma. Recent data clearly demonstrate a key role of adipocytes in the preferential metastasis of ovarian cancer to omentum, indicating their function as an energy source for homing tumour cells (Nieman et al, 2011). These and other data support a significant role of metabolism regulation in tumours and tumour stroma, and suggesting that inclusion of metabolism-regulating agents in cancer therapy should be reexamined with respect to a potential pronounced beneficial effect on the efficacy of the treatment on a system level.

\subsection{TIG3, a target of the MAPK signalling pathway, acts as a tumour suppressor in ovarian cancer cells}

The TIG3 gene was described independently by two groups (Husmann et al., 1998; DiSepio et al., 1998). DiSepio et al. had identified a close homologue of the rat H-rev107-1 gene, named RARRES/TIG3, which was isolated from a differential display approach using Tazarotene-treated human keratinocytes. Tazarotene is a synthetic retinoid, developed for the treatment of psoriasis (Weinstein et al., 1997). Husmann et al. also described a gene closely related to the human $H-r e v 107-1$, named H-REV107-2, isolated during a sequencing project by Merck and the University of Washington. The H-REV107-2 protein differed from RARRES/TIG3 in a longer C-terminal region however; this was recently identified as an artefact (Lotz et al., 2005). Re-sequencing of the H-REV107-2 cDNA construct revealed that the cDNA is identical to the RARRES/TIG3 gene, referred further as TIG3. In addition, a similar sequence cloned from human gastric carcinoma cells was described as RIG1 (Huang et al., 2000). According to sequence comparisons, all proteins are identical except a difference of two amino acids between the proteins deduced from the TIG3 and the RIG1 sequence.

Expression analysis for TIG3 performed on Multiple Tissue Northern Blots and Cancer Profiling Arrays suggested expression of the gene in normal ovary and in many other tissues. Similar to H-REV107-1, TIG3 expression was down-regulated in human ovarian carcinomas and tumour-derived cell lines (DiSepio et al., 1998;Duvic et al., 2000;Shyu et al., 2003;Higuchi et al., 2003;Sturniolo et al., 2003;Lotz et al., 2005) and can be re-expressed upon treatment with IFN $\gamma$ or retinoid and its analogous (Weinstein et al., 1997). 
Up-regulation of TIG3 by IFN $\gamma$ occurs in the same cells in which also H-REV107-1 can be induced by this cytokine. Within the 5' regulatory sequence of the TIG3 gene an IRFresponsive element is present 84 base pairs upstream of the translational start site. However, compared to the related H-REV107-1 gene, TIG3 mRNA levels after IFN $\gamma$-administration follow a different kinetics suggesting that during the IFN $\gamma$-dependent apoptosis, these genes are involved at different stages of the process.

Deregulation of retinoic acid receptors has been involved in ovarian tumours, indicating an essential role of genes targeted by retinoic acid signalling in the prevention of transformation (Benoit et al., 2001;Sun and Lotan, 2002). Furthermore, retinoids represent a promising alternative chemotherapeutic approach for the treatment of late stage ovarian cancer (Zhang et al., 2000;Fields et al., 2007) Consequently, TIG3, involved into retinoic signalling, is likely to be one of the potential mediators for a successful anti-cancer therapy of ovarian carcinomas.

In addition to the retinoic acid responsiveness, we recently detected a negative regulation of TIG3 via an activated MEK-ERK signalling pathway and a positive regulation via IFN- $\gamma$ in ovarian carcinoma cells (Lotz et al., 2005). Thus, like the related H-REV107-1 gene, TIG3 is a target of the oncogenic MEK-ERK signalling pathway. TIG3 itself can dampen the activity of ERK, which suggests an involvement of TIG3 in a negative feedback loop for the control of ERK activity. Inducible and constitutive overexpression of TIG3 cDNA, resulted in growth suppression of A27/80 ovarian carcinoma cells indicating a functional role of the protein in cell growth control (DiSepio et al., 1998; Lotz et al., 2005). However, the mechanisms of ovarian cancer-specific MEK-ERK-dependent TIG3-suppression are unknown.

An important finding was reported by $\mathrm{Ou}$ et al., showing that TIG3 mediates IFN- $\gamma$ dependent down-regulation of HER-2 via regulation of the PI3-kinase pathway (Ou et al., 2008). Using human ovarian carcinoma cell lines OVCAR-3, SKOV-3, and TOV-21G, the group demonstrated an increase of the TIG3 mRNA levels within 2 hours upon administration of IFN- $\gamma$ to the cells. Up-regulation of TIG3 correlated with the downregulation of p185 protein, which could be restored by the application of siRNA against TIG3. A promoter activity assays allowed to demonstrate that TIG3 acts in a HER-2 dependent manner, by a diminishment of the HER-2 activity. Abrogation of HER-2 signalling resulted in a down-regulation of the p185 subunit of the PI3-Kinase. Additionally, VEGF (vascular endothelial growth factor) secretion was regulated in a TIG3-HER-2 dependent manner in a model system. The anti-proliferative, HER-2-inhibiting effect of TIG3 could be abrogated by overexpression of HGR, a member of the neuregulin family activating epidermal growth factor receptor family members and restoring p185 expression (Ou et al., 2008).

This work shows that TIG3 is an important regulator of survival signalling in ovarian carcinomas. Further experiments are necessary, verifying the in vitro observations in animal models of ovarian cancer. Additionally, examination of human ovarian carcinomas and a correlative analysis of TIG3, HER-2 and p185 expression will allow determining the general relevance the observed phenomenon.. Furthermore, due to the co-regulation of TIG3 and HREV107-1 via IFN and MAPK signalling, a reactivation of both genes for therapeutic purposes might exhibit an enhanced anti-apoptotic effect. 


\section{H-REV107-1/HRLS3-driven interplay between PP2A and PKC signal transduction pathways in ovarian carcinomas}

In our previous work, we demonstrated that the class II tumour suppressor H-REV107-1 defined as an enzyme with a phospholipase activity (Jaworski et al., 2009) induces apoptosis in ovarian cancer cells by inhibition of a specific pool of serine/threonine phosphatase PP2A followed by the activation of the atypical PKC $\zeta$ (Nazarenko et al., 2007; Nazarenko et al., 2010).

The PKC family comprises 3 groups of kinases that display very distinct modes of activation and function. The classical PKCs $(\alpha, \beta, \gamma)$ are activated in a calcium-dependent manner through phosphatidylserine (PS) and diacylglycerol (DAG). The novel PKCs $(\delta, \varepsilon, \eta, \theta)$, are also regulated through PS and DAG, but are calcium-independent. Finally, there are the atypical PKCs $(\zeta, 1 / \lambda)$ that require neither calcium nor DAG, but in some cases PS, for activation (Parker and Murray-Rust, 2004;Mackay and Twelves, 2007;Breitkreutz et al., 2007). The different PKC isoenzymes are involved in the regulation of cell survival in normal organs and during tumourigenesis (Shayesteh et al., 1999;Leitges et al., 2001;Martin et al., 2002;Parker and Murray-Rust, 2004;Yin et al., 2005;Moscat et al., 2006). Among the classical PKCs, loss of PKC $\alpha$ in ovarian carcinoma was found to be correlated with increased malignancy (Weichert et al., 2003). While the classical PKC $\alpha$ is down-regulated in ovarian carcinomas, the novel PKC $\theta$ and PKC $\varepsilon$ were found up-regulated in this tumour, yet no functional consequence has been inferred from this deregulation. In addition to the novel $\mathrm{PKC} \theta$ and $\mathrm{PKC} \varepsilon$, also the atypical $\mathrm{PKC}_{1}$ is highly expressed in ovarian carcinomas and acts as a cooperating oncogene with mutant RAS (Zhang et al., 2006).

Recently, we demonstrated that forced expression of H-REV107-1 in ovarian carcinoma cell lines resulted in the inhibition of PP2A activity, re-activation of PP2A target proteins, among them $\mathrm{PKC} \zeta$, and induction of apoptosis (Nazarenko et al., 2007). Importantly, not only tumour cell lines, but also primary tumour cells isolated from the ascites of patients with ovarian carcinomas were sensitive to the treatment with okadaic acid, an inhibitor of PP2A. Induction of apoptosis after okadaic acid treatment was accompanied by the phosphorylation of $\mathrm{PKC} \zeta$, confirming a survival role of PP2A in ovarian cancer, and a potential pro-apoptotic function of PKC $\zeta$. Based on the in vitro cell culture work we analyzed how different members of the PKC family are regulated by H-REV107-1 or by the inhibition of PP2A activity with okadaic acid. Additionally, we verified an impact of the PI3-kinase pathway, a major survival kinase in ovarian carcinoma, in the regulation of PKC.

Analysis of novel PKCs revealed differences at the level of expression and phosphorylation. Thus, treatment with okadaic acid for 48 hours and overexpression of H-REV107-1 led to an increased expression of PKCE. Additionally, H-REV107-1 indirectly induced phosphorylation of the COOH-terminal residue Ser729, shown to enhance the enzymatic activity of PKC $\varepsilon$ (Parekh et al., 2000). This suggests that PKC $\varepsilon$ activity might be partially regulated in an H-REV107-1-dependent manner. Phosphorylation of Thr538 within the activation loop of PKC $\theta$ was elevated after 48 hours of treatment with okadaic acid and the AKT inhibitor LY294002, suggesting a negative but indirect regulation through PP2A and PI-3K. Additionally, Thr538 phosphorylation of PKC $\theta$ was increased in cells expressing HREV107-1, suggesting a potential role of this kinase in H-REV107-1 signalling. The 
phosphorylation of Thr505 located within the activation loop of PKC $\delta$ increased already 15 minutes after the addition of okadaic acid or LY294002, indicating that PKC $\delta$ is directly inactivated by PP2A and PI3K. Although the levels of total PKC $\delta$ seemed to be slightly increased after long-term okadaic acid and LY294002 treatment, the phosphorylation was strongly diminished. H-REV107-1 negatively regulated the expression of PKCD, supporting the finding that PKC $\delta$ is not involved in H-REV107-1-dependent cell death. Expression of atypical PKCl was increased following 48 hours of treatment with okadaic acid, but neither phosphorylation nor total levels were affected by H-REV107-1.

To correlate phosphorylation of kinases in the activation site and their intracellular kinase activity, we applied in vitro kinase assay described in detail elsewhere (Nazarenko et al., 2010) and measured direct changes in the activity of PKCs upon okadaic acid treatment. A significant elevation of the PKC $\theta$ and PKC $\varepsilon$ activity was detected 24 hours after okadaic acid incubation, confirming that these PKCs, although not known to be direct PP2A targets, are negatively regulated by PP2A signalling in OVCAR-3 cells.

As inhibition of PP2A is required for H-REV107-1-dependent apoptosis, we next asked if these kinases might be involved in H-REV107-1-induced cell death and tested if the abrogation of PKC $\theta$ and PKC $\varepsilon$ activity impairs the proapoptotic function of H-REV107-1. OVCAR-3 cells were transfected either with the H-REV107-1 expression vector or with a control plasmid. Twelve hours later, the PKCO- and PKCE-specific peptides were added. Caspase-3 cleavage was tested after 48 hours using Western blot analysis. H-REV107-1 expression resulted in the induction of caspase- 3 cleavage, which was however not altered after peptide applications. Additionally, PKC $\theta$-specific peptide treatment of control cells revealed a weak toxic effect. This result suggests that although PKC $\varepsilon$ and PKC $\theta$ are clearly activated in a PP2A and H-REV107-1-dependent manner, they are not essential for the HREV107-1 proapoptotic activity in OVCAR-3 cells.

An important finding was that the atypical PKC $\zeta$ is uncoupled from the PI3K pathway in ovarian cancer cells and is more likely to be a PP2A target. This is in contrast to the situation in the majority of normal and malignant tissues, in which PKC $\zeta$ functions as an insulin-dependent PI3K effector. Importantly, overexpression of wild type H-REV107-1, but not of its PP2A interaction-deficient mutant, led to PKC phosphorylation, suggesting a direct link between the ability of H-REV107-1 to inhibit PP2A and the activation of PKC了.

Electroporation of the ovarian carcinoma cells with PKC $\mathrm{P}$-expression plasmid demonstrated that high levels of this kinase are sufficient to induce apoptosis. In our work we demonstrated an increase of the sub-G1 cell population and caspase-3 cleavage. Molecular mechanisms by mean of which PKC $\zeta$ induces apoptosis remained elusive and need further investigations. A recent work of Peng et al. might provide an additional hint for the mechanisms of PKC -dependent apoptosis (Chen et al., 2008). Using a mouse model, the authors demonstrated that PKC $\zeta$ directly interacts with ERK1/2 in Kupffer cells, mediating a translocation of NF-kB into the nucleus and inducing its activity. The novelty of this finding is a direct link between PKC $\zeta, E K R 1 / 2$ and NF-kB. Consistently, a cross-talk between NF-kB and PKC $\zeta$ is well- characterised for many systems (Moscat et al., 2001;Moscat and az-Meco, 2011). Next, a potential interaction between PKC $\zeta$, ERK1/2, and NF-kB in ovarian cancer cells should be verified. A hypothetical scheme of PKC apoptotic cascade and cross-talk with other pathways is represented on the Fig. 5. 


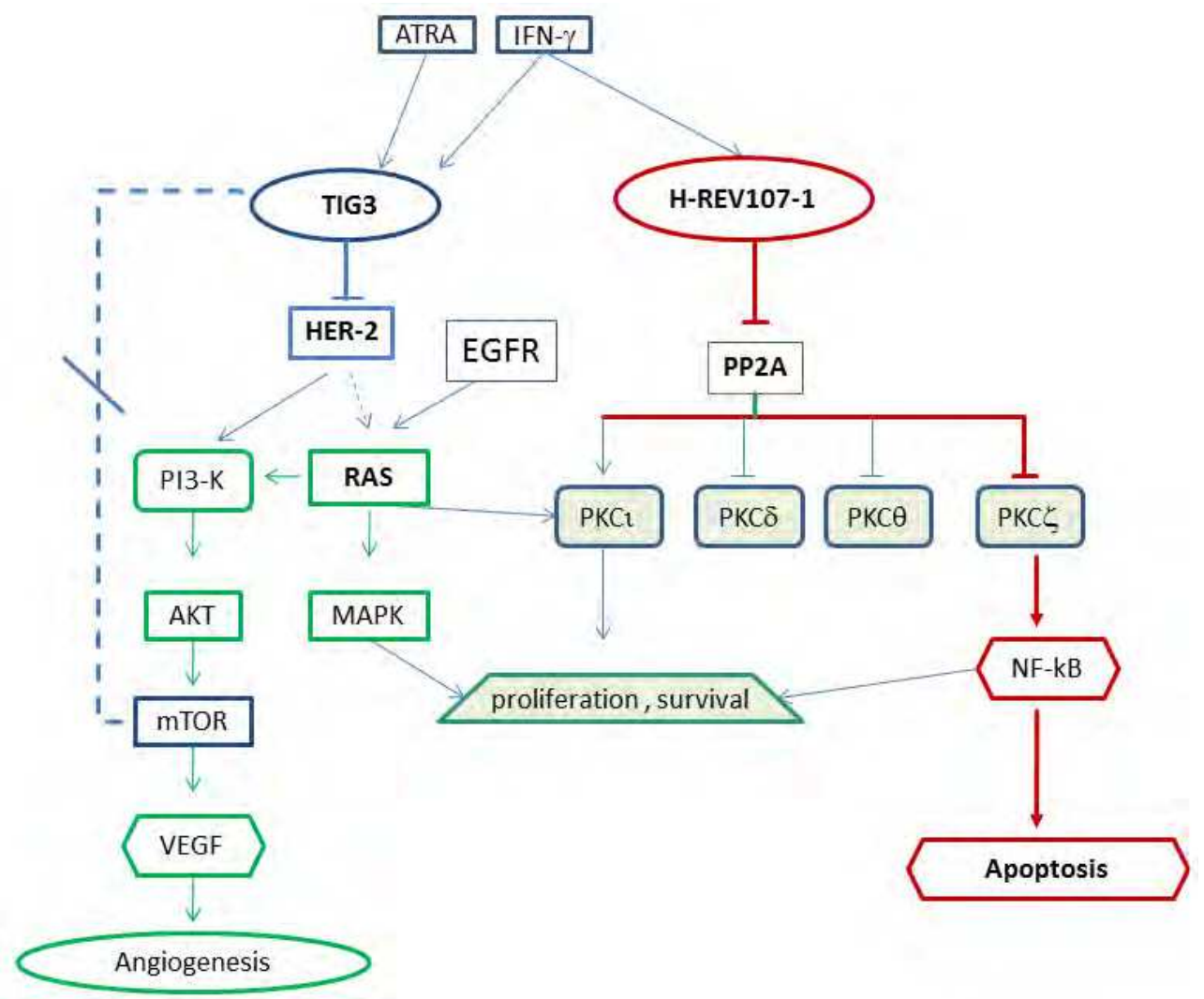

Fig. 5. Hypothetical scheme of the pro-apoptotic signal transduction network in ovarian cancer cells of two members of the HRS protein family, the H-REV107-1 (showed with red arrows) and TIG3 (showed with blue arrows). Both family members can be activated by IFN $\gamma$, whereas TIG3 can be additionally activated by ATRA. TIG3 mediates inhibition of HER-2, mediating herewith suppression of angiogenesis. H-REV107-1 inhibits PP2A, mediating activation of PKC $\zeta$. PKC $\zeta$ functions as a major mediator of H-REV107-1-mediated cell death and is sufficient to induce apoptosis in a subset ovarian carcinoma cells, sensitive to the H-REV107-1--mediated apoptosis.

\section{Receptor kinase pathway profiling in ovarian cancer cells}

We applied the RPPA (reverse phase protein array) technique to define a potential regulation of PKCs by epidermal growth factor receptor inhibition. Earlier work on the HREV107-1 tumour suppressor, an inhibitor of PP2A, has demonstrated that H-REV1071-1 is lost in a significant portion of ovarian tumours.

As a class II tumour suppressor gene, H-REV107-1 expression was reconstituted upon IFN $\gamma$ treatment and MAPK inhibition and was able to induce specific phosphorylation of atypical PKC and the induction of apoptosis. These observations suggested to us that interference with oncogenic pathways might also have some impact on PKC isoform expression and/or 
activation. Therefore, we asked whether PKC $\zeta$ phosphorylation, which is necessary for apoptosis induction in ovarian cancer cells, might also be regulated by inhibitors and therapeutic agents that target mitogenic and survival pathways. The most prominent candidates for such an approach appeared to be the family of epidermal growth factor receptors (EGFR), whose members are frequently mutated and activated in human malignancies, and specific inhibitors are used for the treatment of ovarian carcinomas (De Marinis et al., 2002;Blank et al., 2005).

We performed a reverse phase protein array analysis (RPPA) of OVCAR-3 cells treated with the EGFR inhibitors Cetuximab and Gefitinib/Iressa, and tested the expression and phosphorylation of PKC $\zeta$ and the expression of PKC $\alpha$ and PKC $\delta$ with antibodies established for this approach. To test if other signalling cascades are similarly affected following inhibition of EGFR signalling, we applied antibodies against phosphorylated AKT and ERK proteins and against the Bad protein.

This analysis showed an increased protein level of PKC $\zeta$ and of its phosphorylated form 24 hours after the treatment with EGFR inhibitors. The RPPA analysis of ERK, AKT and Bad proteins revealed a moderate effect of EGFR inhibition on the phosphorylation status of these proteins and their expression. While total levels of Akt/PKB and phosphorylation of Bad Ser112 were unchanged, Akt/PKC and ERK phosphorylation were moderately increased after application of inhibitors. In addition to PKC $\zeta$, RPPA analysis also revealed elevated levels of PKC $\alpha$ and PKC $\delta$ following incubation with the EGFR inhibitors, suggesting a role of these kinases in EGFR downstream signalling.

Our experimental data obtained through profiling with reverse phase protein arrays revealed that application of Cetuximab or Gefitinib to OVCAR-3 cells induced only a moderate effect on MAPK and PI3K signalling, and had no effect onto cell growth. This suggests that specific targeting of EGFR is not sufficient to switch the survival program to an apoptotic program in these cells. In addition, EGFR inhibition led to a transient activation of PKC $\zeta$ and to an up-regulation of PKC $\alpha$ and PKC $\delta$. Neither PKC $\alpha$ nor PKC $\delta$ seem to play a crucial role in apoptosis induction in the cell lines tested, while we provided clear evidence for an involvement of PKC $\zeta$ in the induction of apoptosis. The transient activation of PKC $\zeta$ following EGFR interference was not sufficient to induce apoptosis. Therefore, the inhibition of oncogenic tyrosine kinase receptors might be a prerequisite for full or partial reconstitution of the players involved in apoptosis, but an additional trigger such as chemotherapy might be necessary to actually execute the death program (Nazarenko et al., 2010).

\section{Conclusion}

This chapter describes the impact of a family of tumour suppressor proteins, and the specific PKC $\zeta$-mediated signalling on apoptosis induction in ovarian cancer. The genes encoding $\mathrm{H}-$ REV107-1/HRSL3 and TIG3 both act as tumour suppressor genes. While the functional impact of TIG3 is still somewhat elusive, H-REV107-1 governs the decision between survival and apoptosis. Of major importance for the future research is the newly described function of H-REV107-1 and its related proteins, being phospholipases. This function indicates a specific role of lipid metabolism in the control of transformation and potentially tumour progression. 
Furthermore, high expression levels of PKC $\zeta$ and a correlation with poor prognosis were observed in human ovarian carcinoma samples and only the activation of endogenous PKC $\zeta$ by okadaic acid or by the HRSL3 tumour suppressor, correlated with the induction of apoptosis in primary and immortalized ovarian carcinoma cells. This suggests a potentially inaccessible pro-apoptotic action of this kinase, which might be negatively regulated by activated tyrosine kinase receptors in ovarian cancer. In future research, identification of yet unknown substrates of the members of the HRS family will support current knowledge on the mechanisms of their pro-apoptotic function. Possibly, new aspects of functions, opening novel horizons in the therapy of ovarian cancer therapy, will be developed.

\section{Acknowledgment}

We thank Andreas Weihe and Uwe Richter (Institute of Genetics, Humboldt University, Berlin, Germany) for their help in the phylogenetic analysis of the HRS and HRS-related families. Steffen Reich (Institute of Pathology, Charité, Berlin, Germany, for the analysis of H-REV107-1 human promoter.

\section{References}

Akiyama, H., Y. Hiraki, M. Noda, C. Shigeno, H. Ito, and T. Nakamura, 1999, Molecular cloning and biological activity of a novel Ha-Ras suppressor gene predominantly expressed in skeletal muscle, heart, brain, and bone marrow by differential display using clonal mouse EC cells, ATDC5: J.Biol.Chem., v. 274, no. 45, p. 32192-32197.

Alessi, D. R., Y. Saito, D. G. Campbell, P. Cohen, G. Sithanandam, U. Rapp, A. Ashworth, C. J. Marshall, and S. Cowley, 1994, Identification of the sites in MAP kinase kinase-1 phosphorylated by p74raf-1: EMBO J., v. 13, no. 7, p. 1610-1619.

Anantharaman, V., and L. Aravind, 2003, Evolutionary history, structural features and biochemical diversity of the NlpC/P60 superfamily of enzymes: Genome Biol., v. 4, no. 2.

Bartel, D. P., 2004, MicroRNAs: genomics, biogenesis, mechanism, and function: Cell, v. 116, no. 2, p. 281-297.

Benoit, G. R. et al., 2001, Autonomous rexinoid death signaling is suppressed by converging signaling pathways in immature leukemia cells: Mol.Endocrinol., v. 15, no. 7, p. 1154-1169.

Blank, S. V., R. Chang, and F. Muggia, 2005, Epidermal growth factor receptor inhibitors for the treatment of epithelial ovarian cancer: Oncology (Williston.Park), v. 19, no. 4, p. 553-559.

Breitkreutz, D., L. Braiman-Wiksman, N. Daum, M. F. Denning, and T. Tennenbaum, 2007, Protein kinase C family: on the crossroads of cell signaling in skin and tumor epithelium: J.Cancer Res.Clin.Oncol., v. 133, no. 11, p. 793-808.

Brown, L. A. et al., 2008, Amplification of 11q13 in ovarian carcinoma: Genes Chromosomes.Cancer, v. 47, no. 6, p. 481-489.

Chen, Y., Z. Liu, S. Liang, X. Luan, F. Long, J. Chen, Y. Peng, L. Yan, and J. Gong, 2008, Role of Kupffer cells in the induction of tolerance of orthotopic liver transplantation in rats: Liver Transpl., v. 14, no. 6, p. 823-836. 
Choi, J. H., J. J. Sheu, B. Guan, N. Jinawath, P. Markowski, T. L. Wang, and I. Shih, 2009, Functional analysis of 11q13.5 amplicon identifies Rsf-1 (HBXAP) as a gene involved in paclitaxel resistance in ovarian cancer: Cancer Res., v. 69, no. 4, p. 14071415.

De Marinis, F., F. Nelli, and G. D'Auria, 2002, EGFR inhibitors: clinical results: Suppl Tumori, v. 1, no. 6, p. S5-S6.

DiSepio, D., C. Ghosn, R. L. Eckert, A. Deucher, N. Robinson, M. Duvic, R. A. S. Chandraratna, and S. Nagpal, 1998, Identification and characterization of a retinoid-induced class II tumor suppressor growth regulatory gene: Proc.Natl.Acad.Sci.U.S.A., v. 95, no. 25, p. 14811-14815.

Duvic, M. et al., 2000, Expression of a retinoid-inducible tumor suppressor, Tazaroteneinducible gene-3, is decreased in psoriasis and skin cancer: Clin.Cancer Res., v. 6, no. 8, p. 3249-3259.

Duvic, M., X. Ni, R. Talpur, K. Herne, C. Schulz, D. Sui, S. Ward, A. Joseph, and P. Hazarika, 2003, Tazarotene-induced gene 3 is suppressed in basal cell carcinomas and reversed in vivo by tazarotene application: J.Invest.Dermatol., v. 121, no. 4, p. $902-$ 909.

Faratian, D., I. Um, D. S. Wilson, P. Mullen, S. P. Langdon, and D. J. Harrison, 2011, Phosphoprotein pathway profiling of ovarian carcinoma for the identification of potential new targets for therapy: Eur.J.Cancer, v. 47, no. 9, p. 1420-1431.

Fields, A. L., D. R. Soprano, and K. J. Soprano, 2007, Retinoids in biological control and cancer: J.Cell Biochem., v. 102, no. 4, p. 886-898.

Forbes, S. A. et al., 1997, Mapping of the gene encoding the B56 beta subunit of protein phosphatase 2A (PPP2R5B) to a $0.5-\mathrm{Mb}$ region of chromosome $11 \mathrm{q} 13$ and its exclusion as a candidate gene for multiple endocrine neoplasia type 1 (MEN1): Hum.Genet., v. 100, no. 3-4, p. 481-485.

Gansler TS, Hardman W, Hunt DA, Schaffel S, Hennigar RA, 1997, Increased expression of fatty acid synthase (OA-519) in ovarian neoplasms predicts shorter survival: Hum Pathol., v. 28, p. 686-692

Hajnal, A., R. Klemenz, and R. Schafer, 1994, Subtraction Cloning of H-Rev107, A Gene Specifically Expressed in H-Ras Resistant Fibroblasts: Oncogene, v. 9, no. 2, p. 479490.

Higuchi, E., R. A. Chandraratna, W. K. Hong, and R. Lotan, 2003, Induction of TIG3, a putative class II tumor suppressor gene, by retinoic acid in head and neck and lung carcinoma cells and its association with suppression of the transformed phenotype: Oncogene, v. 22, no. 30, p. 4627-4635.

Huang, S. L., R. Y. Shyu, M. Y. Yeh, and S. Y. Jiang, 2000, Cloning and characterization of a novel retinoid-inducible gene 1(RIG1) deriving from human gastric cancer cells: Mol.Cell.Endocrinol., v. 159, no. 1-2, p. 15-24.

Huang, S. L., R. Y. Shyu, M. Y. Yeh, and S. Y. Jiang, 2002, The retinoid-inducible gene I: Effect on apoptosis and mitogen-activated kinase signal pathways: Anticancer Res., v. 22, no. 2A, p. 799-804.

Hughes, P. J., and G. Stanway, 2000, The 2A proteins of three diverse picornaviruses are related to each other and to the H-rev107 family of proteins involved in the control of cell proliferation: J.Gen.Virol., v. 81, no. Pt 1, p. 201-207. 
Husmann, K., C. Sers, E. Fietze, A. Mincheva, P. Lichter, and R. Schafer, 1998, Transcriptional and translational downregulation of H-REV107, a class II tumour suppressor gene located on human chromosome 11q11-12: Oncogene, v. 17, no. 10, p. 1305-1312.

Ito, H., H. Akiyama, C. Shigeno, and T. Nakamura, 2001, Isolation, characterization, and chromosome mapping of a human A-C1 Ha-Ras suppressor gene (HRASLS): Cytogenet.Cell Genet., v. 93, no. 1-2, p. 36-39.

Jaworski, K. et al., 2009, AdPLA ablation increases lipolysis and prevents obesity induced by high-fat feeding or leptin deficiency: Nat.Med., v. 15, no. 2, p. 159-168.

Kjeldgaard, M., J. Nyborg, and B. F. Clark, 1996, The GTP binding motif: variations on a theme: FASEB J., v. 10, no. 12, p. 1347-1368.

Leitges, M. et al., 2001, Targeted disruption of the zetaPKC gene results in the impairment of the NF-kappaB pathway: Mol.Cell, v. 8, no. 4, p. 771-780.

Lotz, K., T. Kellner, M. Heitmann, I. Nazarenko, A. Noske, A. Malek, A. Gontarewicz, R. Schafer, and C. Sers, 2005, Suppression of the TIG3 tumor suppressor gene in human ovarian carcinomas is mediated via mitogen-activated kinase-dependent and -independent mechanisms: Int.J.Cancer, v. 116, no. 6, p. 894-902.

Mackay, H. J., and C. J. Twelves, 2007, Targeting the protein kinase C family: are we there yet?: Nat.Rev.Cancer, v. 7, no. 7, p. 554-562.

Martin, P., A. Duran, S. Minguet, M. L. Gaspar, M. T. Diaz-Meco, P. Rennert, M. Leitges, and J. Moscat, 2002, Role of zeta PKC in B-cell signaling and function: EMBO J., v. 21, no. 15 , p. 4049-4057.

McCubrey, J. A. et al., 2007, Targeting the RAF/MEK/ERK, PI3K/AKT and p53 pathways in hematopoietic drug resistance: Adv.Enzyme Regul., v. 47, p. 64-103.

Menendez JA, Vellon L, Mehmi I, et al., 2004, Inhibition of fatty acid synthase (f a s) suppresses h e $\mathrm{r} 2$ / neu (ErbB-2) oncogene overexpression in cancer cells. Proc Natl Acad Sci U S A, v. 101, p 10715-10720.

Moscat, J., and M. T. az-Meco, 2011, Fine tuning NF-kappaB: new openings for PKC-zeta: Nat.Immunol., v. 12, no. 1, p. 12-14.

Moscat, J., L. Sanz, P. Sanchez, and M. T. az-Meco, 2001, Regulation and role of the atypical $\mathrm{PKC}$ isoforms in cell survival during tumor transformation: Adv.Enzyme Regul., v. 41, p. 99-120.

Moscat, J., P. Rennert, and M. T. Diaz-Meco, 2006, PKCzeta at the crossroad of NF-kappaB and Jak1/Stat6 signaling pathways: Cell Death.Differ., v. 13, no. 5, p. 702-711.

Nazarenko, I. et al., 2010, Atypical protein kinase C zeta exhibits a proapoptotic function in ovarian cancer: Mol.Cancer Res., v. 8, no. 6, p. 919-934.

Nazarenko, I., R. Schafer, and C. Sers, 2007, Mechanisms of the HRSL3 tumor suppressor function in ovarian carcinoma cells: J.Cell Sci., v. 120, no. Pt 8, p. 1393-1404.

Nicosia, S. V., W. Bai, J. Q. Cheng, D. Coppola, and P. A. Kruk, 2003, Oncogenic pathways implicated in ovarian epithelial cancer: Hematol.Oncol.Clin.North Am., v. 17, no. 4, p. 927-943.

Ou, C. C., S. C. Hsu, Y. H. Hsieh, W. L. Tsou, T. C. Chuang, J. Y. Liu, and M. C. Kao, 2008, Downregulation of HER2 by RIG1 involves the PI3K/Akt pathway in ovarian cancer cells: Carcinogenesis, v. 29, no. 2, p. 299-306.

Parekh, D. B., W. Ziegler, and P. J. Parker, 2000, Multiple pathways control protein kinase C phosphorylation: EMBO J., v. 19, no. 4, p. 496-503. 
Parker, P. J., and J. Murray-Rust, 2004, PKC at a glance: J.Cell Sci., v. 117, no. Pt 2, p. 131-132. Ren, X., J. Lin, C. Jin, and B. Xia, 2010a, 1H, 13C and 15N resonance assignments of human H-REV107 N-terminal domain: Biomol.NMR Assign., v. 4, no. 2, p. 175-178.

Ren, X., J. Lin, C. Jin, and B. Xia, 2010b, Solution structure of the N-terminal catalytic domain of human H-REV107--a novel circular permutated NlpC/P60 domain: FEBS Lett., v. 584, no. 19 , p. $4222-4226$.

Roberts, P. J., and C. J. Der, 2007, Targeting the Raf-MEK-ERK mitogen-activated protein kinase cascade for the treatment of cancer: Oncogene, v. 26, no. 22, p. 3291-3310.

Roder, K., M. J. Latasa, and H. S. Sul, 2002, Silencing of the mouse H-rev107 gene encoding a class II tumor suppressor by CpG methylation: J.Biol.Chem., v. 277, no. 34, p. 30543-30550.

Sager, R., 1997, Expression genetics in cancer: shifting the focus from DNA to RNA: Proc.Natl.Acad.Sci.U.S.A, v. 94, no. 3, p. 952-955.

Schafer, R., and C. Sers, 2010, RAS oncogene-mediated deregulation of the transcriptome: From molecular signature to function: Adv.Enzyme Regul..

Sers, C. et al., 2002, The class II tumour suppressor gene H-REV107-1 is a target of interferon-regulatory factor- 1 and is involved in IFNgamma-induced cell death in human ovarian carcinoma cells: Oncogene, v. 21, no. 18, p. 2829-2839.

Sers, C., U. Emmenegger, K. Husmann, K. Bucher, A. C. Andres, and R. Schafer, 1997, Growth-inhibitory activity and downregulation of the class II tumor-suppressor gene H-rev107 in tumor cell lines and experimental tumors: J.Cell Biol., v. 136, no. 4, p. 935-944.

Shayesteh, L. et al., 1999, PIK3CA is implicated as an oncogene in ovarian cancer: Nat.Genet., v. 21, no. 1, p. 99-102.

Shyu, R. Y., S. Y. Jiang, J. M. Chou, Y. L. Shih, M. S. Lee, J. C. Yu, P. C. Chao, Y. J. Hsu, and S. W. Jao, 2003, RARRES3 expression positively correlated to tumour differentiation in tissues of colorectal adenocarcinoma: Br.J.Cancer, v. 89, no. 1, p. 146-151.

Siegrist, S., C. Feral, M. Chami, B. Solhonne, M. G. Mattei, E. Rajpert-De Meyts, G. Guellaen, and F. Bulle, 2001, hH-Rev107, a class II tumor suppressor gene, is expressed by post-meiotic testicular germ cells and CIS cells but not by human testicular germ cell tumors: Oncogene, v. 20, no. 37, p. 5155-5163.

Song Y., Wilkins P., Hu W., et al., 2007, Inhibition of calcium-independent phospholipase A2 suppresses proliferation and tumorigenicity of ovarian carcinoma cells: Biochem J, v 406, p.427-436.

Strimmer, K., and A. von Haeseler, 1997, Likelihood-mapping: a simple method to visualize phylogenetic content of a sequence alignment: Proc.Natl.Acad.Sci.U.S.A, v. 94, no. 13, p. 6815-6819.

Sturniolo, M. T., S. R. Dashti, A. Deucher, E. A. Rorke, A. M. Broome, R. A. S. Chandraratna, T. Keepers, and R. L. Eckert, 2003, A novel tumor suppressor protein promotes keratinocyte terminal differentiation via activation of type I transglutaminase: J.Biol.Chem., v. 278, no. 48, p. 48066-48073.

Sun, S. Y., and R. Lotan, 2002, Retinoids and their receptors in cancer development and chemoprevention: Crit Rev.Oncol.Hematol., v. 41, no. 1, p. 41-55.

Tanja, M.S., Khan, M.A., Song, Y. 2010, Association of lipid metabolism with ovarian cancer. Current Oncology., v. 17., no. 3, p. 6-11. 
Weichert, W., V. Gekeler, C. Denkert, M. Dietel, and S. Hauptmann, 2003, Protein kinase C isoform expression in ovarian carcinoma correlates with indicators of poor prognosis: Int.J.Oncol., v. 23, no. 3, p. 633-639.

Weinstein, G. D. et al., 1997, Tazarotene gel, a new retinoid, for topical therapy of psoriasis: vehicle-controlled study of safety, efficacy, and duration of therapeutic effect: J.Am.Acad.Dermatol., v. 37, no. 1, p. 85-92.

Weinstein, I. B., 1987, Growth factors, oncogenes, and multistage carcinogenesis: J.Cell Biochem., v. 33, no. 3, p. 213-224.

Yin, L., N. Bennani-Baiti, and C. T. Powell, 2005, Phorbol ester-induced apoptosis of C4-2 cells requires both a unique and a redundant protein kinase $C$ signaling pathway: J.Biol.Chem., v. 280, no. 7, p. 5533-5541.

Zhang, D., W. F. Holmes, S. Wu, D. R. Soprano, and K. J. Soprano, 2000, Retinoids and ovarian cancer: J.Cell Physiol, v. 185, no. 1, p. 1-20.

Zhang, L. et al., 2006, Integrative genomic analysis of protein kinase C (PKC) family identifies PKCiota as a biomarker and potential oncogene in ovarian carcinoma: Cancer Res., v. 66, no. 9, p. 4627-4635.
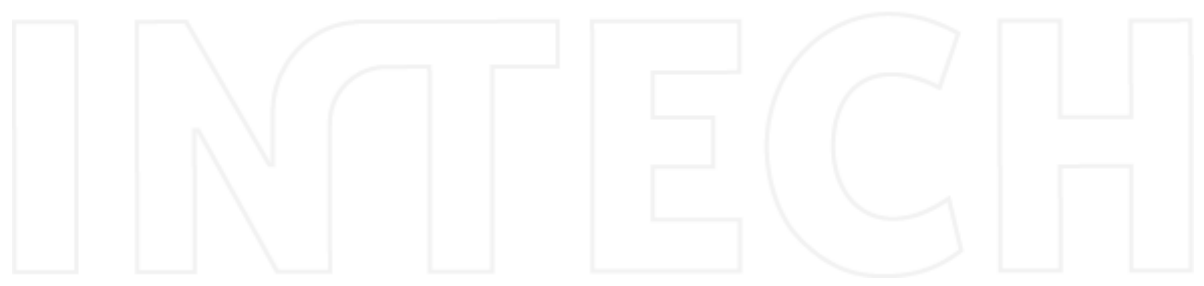


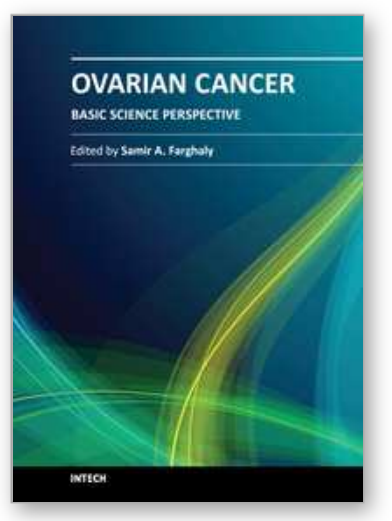

\author{
Ovarian Cancer - Basic Science Perspective \\ Edited by Dr. Samir Farghaly
}

ISBN 978-953-307-812-0

Hard cover, 406 pages

Publisher InTech

Published online 17, February, 2012

Published in print edition February, 2012

Worldwide, Ovarian carcinoma continues to be responsible for more deaths than all other gynecologic malignancies combined. International leaders in the field address the critical biologic and basic science issues relevant to the disease. The book details the molecular biological aspects of ovarian cancer. It provides molecular biology techniques of understanding this cancer. The techniques are designed to determine tumor genetics, expression, and protein function, and to elucidate the genetic mechanisms by which gene and immunotherapies may be perfected. It provides an analysis of current research into aspects of malignant transformation, growth control, and metastasis. A comprehensive spectrum of topics is covered providing up to date information on scientific discoveries and management considerations.

\title{
How to reference
}

In order to correctly reference this scholarly work, feel free to copy and paste the following:

Christine Sers, Reinhold Schafer and Irina Nazarenko (2012). Apoptosis Pathways in Ovarian Cancer, Ovarian Cancer - Basic Science Perspective, Dr. Samir Farghaly (Ed.), ISBN: 978-953-307-812-0, InTech, Available from: http://www.intechopen.com/books/ovarian-cancer-basic-science-perspective/apoptosis-pathwayprofiling-in-ovarian-cancer

\section{INTECH}

open science | open minds

\section{InTech Europe}

University Campus STeP Ri Slavka Krautzeka 83/A 51000 Rijeka, Croatia Phone: +385 (51) 770447 Fax: +385 (51) 686166 www.intechopen.com

\section{InTech China}

Unit 405, Office Block, Hotel Equatorial Shanghai

No.65, Yan An Road (West), Shanghai, 200040, China

中国上海市延安西路65号上海国际贵都大饭店办公楼405单元

Phone: +86-21-62489820

Fax: +86-21-62489821 